\author{
Voix plurielles \\ Volume 5, Numéro 1 : mai 2008
}

Frédéric Marteau

\title{
Écrire, habiter, penser Autour de l'oeuvre de Thomas Bernhard
}

Citation MLA : Marteau, Frédéric. «Écrire, habiter, penser. Autour de l'oeuvre de Thomas Bernhard.»Voix plurielles 5.1 (mai 2008).

(C) Voix plurielles, revue électronique de l'APFUCC 2008. 


\title{
Écrire, habiter, penser Autour de l'œuvre de Thomas Bernhard
}

\author{
Frédéric Marteau \\ Université Paris 8
}

Mai 2008

\section{Résumé}

Les romans et récits de Thomas Bernhard tournent souvent autour d'un lieu spécifique ; plus généralement, la thématique de l'habiter hante son œuvre; car penser, c'est-à-dire aussi écrire, nécessite un lieu de recueillement. Mais ce lieu de pensée s'avère être un lieu d'enfermement, qui mène à la destruction et à l'anéantissement. Digne héritier de Kafka, Bernhard entremêle différents types de constructions : des constructions d'habitats, des constructions de pensées et des constructions verbales. Tentative paradoxale de construction/destruction de et dans la langue, l'œuvre de Bernhard éclaire les relations entre la maison et le livre, l'écrire et l'habiter, l'extinction des lieux et le surgissement paradoxal de l'œuvre.

Lorsque l'entendement est rigoureux, la pensée est des plus impitoyables et des plus claires, dit Oehler, nous sommes obligés de dire dans le délai le plus bref à propos de tout, que c'est insupportable et effroyable. L'art consiste donc sans aucun doute à supporter l'insupportable et à ne pas ressentir ce qui est effroyable comme tel, c'est-à-dire effroyable. Il va de soi que cet art doit être défini comme le plus difficile qui soit. L'art d'exister contre les faits, dit Oehler, est l'art le plus difficile qui soit. Exister contre les faits signifie, dit Oehler, exister contre l'insupportable et l'effroi. Si nous n'existons pas constamment contre mais seulement avec les faits, dit Oehler, nous succombons dans les plus brefs délais.

(Marcher 363-4)

L'œuvre de Thomas Bernhard se présente comme un ensemble cohérent d'ouvrages qui reprennent et développent les mêmes thématiques et les mêmes motifs. Rarement un auteur aura à ce point travaillé un style et un propos restreints - pour ne pas dire limités - à quelques traits marquants, un style et un propos sidérés par le ressassement, le va-et-vient monologique, la répétition rythmée ou musicale de propositions qui creusent et retracent les mêmes sillons, sans jamais envisager ouvertement la possibilité d'en sortir. Euvre du trait et du re-trait (du rittrato, de l'autoportrait), qui dessine une figure littéraire singulière hantée par une double nécessité : 
penser (denken), c'est-à-dire aussi bâtir, construire (bauen) ou écrire (schreiben), mais également hantée par une impossibilité : l'impossibilité d'habiter un lieu (wohnen), ou, pour reprendre une célèbre formule de Hölderlin, «d'habiter poétiquement » sur cette terre. Cette triple hantise, de penser, d'écrire et d'habiter, se situe dans une modernité historico-littéraire qui se devait de combattre tout un héritage : celui d'un pays (l'Autriche), celui d'un passé (le nazisme et sa conception du «sang et du sol », du Blut und Boden) et celui d'une enfance personnelle marquée par l'absence paternelle, l'abandon maternel et les assauts de la maladie ${ }^{1}$. Il s'agit alors pour Bernhard de proposer une nouvelle forme de récit qui sache affronter (affirmer - bejahen) la négativité qu'évitent ses contemporains, de construire une œuvre à partir de sa propre ruine et de son propre désœuvrement, et de penser la «véritable crise de l'habitation » d'une manière toute différente de celle qu'Heidegger développe dans sa conférence de 1951, «Bâtir habiter penser (Bauen wohnen denken) » (Heidegger 170-93). L'écrivain choisit le plus souvent une attitude contradictoire et adopte une position contre, la seule possible : écrire contre, penser contre, habiter dans la contra-diction. Au final (ou : à la limite), cette entreprise-contre (cette actioncontre - cette contraction ou «correction ») développe un art de l'exagération (Übertreibung) ${ }^{2}$ qui amène l'œuvre à exister malgré tout, à se construire sur ses propres ruines et ses propres impossibilités.

\section{Thomas Bernhard et l'habitat : architecture d'une œuvre}

Les récits de Thomas Bernhard se plaisent à situer les personnages dans des lieux spécifiques. Refusant le plus souvent la description traditionnelle ${ }^{3}$, ces lieux n'existent que sous le signe de quelques caractéristiques, de quelques détails, et se résument même parfois à leur seul toponyme. Le château de Hochgobernitz, la Plâtrière de Sicking, Altensam, la mansarde de la construction Höller dans la gorge de l'Aurach, le château de Wolfsegg (et sa Villa des enfants), etc. : chaque récit est émaillé de noms de lieux, noms de ville ou lieux-dits; des noms propres qui obsèdent les personnages qui y vivent, ou qui y ont des relations. Dès Amras, les lieux jouent un rôle essentiel dans le récit: deux frères, ayant survécu au suicide familial organisé par leurs parents, se réfugient dans une tour («emblème d'Amras »), sur les conseils de leur oncle; à la mort de Walter, le narrateur s'isole dans la maison forestière d'Aldrans. Le lieu de vie devient un élément transcendantal, la condition de possibilité d'une réflexion, d'un travail, d'une prise de conscience :

Dans la tour, nous avions soudain pris pleinement conscience des ténèbres les plus épaisses, par instants... de la stupidité des possibilités... c'est dans la tour que nous avions pris conscience de nous-mêmes, c'est là que nous nous regardions pour la première fois, de l'extérieur et de l'intérieur... (Amras 34)

Une fois réfugié dans la forêt, le narrateur fait encore davantage l'épreuve de l'habiter; il distingue par exemple deux types d'habitants définissant deux modes d'habiter: ceux qui habitent à la ferme (et « qui vont dans la forêt avec des guêtres ») et ceux qui n'habitent pas à la ferme. "Comme si ceux qui habitent à la ferme et ceux qui n'habitent pas à la ferme appartenaient à des mondes différents » (Amras 58). Nous sommes en fait conditionnés par notre 
environnement, et cette constatation sera toujours à l'origine des passions des personnages bernhardiens; un environnement d'ailleurs fortement autrichien, comme aime à le souligner l'écrivain, un beau pays étouffant qui s'avère le plus souvent inhabitable, causant les maux les plus terribles, les plus mortels. Après le peintre Strauch de Gel, le narrateur d'Amras en fait le constat épouvantable :

Notre existence, cela ne fait aucun doute, a été suscitée par ce paysage et cet air du Tyrol, qui délabrent les systèmes nerveux et les systèmes cérébraux les plus sensibles, les plus inflammables... (71)

Nous, Walter et moi, avions toujours été trompés, par une composition de l'air désespérée, par un galvanisme patriarcal inhumain, provoqué par les hauteurs et les abîmes perfides de sa nature d'architecture... Combien de nos talents aurions-nous pu épanouir en nous jusqu'à une ampleur surprenante, si nous n'étions pas nés et n'avions pas grandi au Tyrol! (73)

Comment habiter un tel lieu inhabitable, à l'air irrespirable ? La question sera déclinée dans tous les ouvrages suivants, sur le mode d'une exagération tragi-comique toujours active. C'est pourquoi dans chacun de ses récits, Bernhard placera la maison, le lieu d'habitation, au centre des préoccupations des personnages, apparaissant tantôt comme une riposte ou une alternative à l'agression ambiante (géographique, nationale, étatique), tantôt comme sa manifestation la plus impitoyable. La maison sera dès lors tour à tour un lieu de protection ou un lieu d'étouffement, comme l'ont peut-être symbolisé, pour Bernhard, les galeries anti-bombardement (Stollen) qu'il a connu à Salzbourg pendant la guerre et dont il parle dans L'Origine ou dans ce dernier texte d'Événements où « Le survivant note »:

Vers la fin de la guerre, on creuse des galeries dans les deux collines de la ville, dans lesquelles les gens affluent, parce que l'anéantissement les menace. C'est uniquement parce qu'ils entrent dans les galeries qu'ils ont la vie sauve. D'abord, ils n'osent pas se risquer à la lumière du jour. C'est seulement en hésitant qu'ils laissent franchir les portes à ceux qui leur semblent sans valeur et faibles, aux enfants aussi pour finir, et l'après-midi ils quittent tous en silence les galeries, où beaucoup d'entre eux sont morts étouffés, parce qu'ils avaient trop peu d'oxygène. . . Mais une fois que la guerre est finie, il se passe quelque chose que personne ne parvient à comprendre : ils ne murent pas les galeries, ils y vont au contraire, comme ils en ont pris l'habitude. Tous les jours à la même heure. Aussi longtemps qu'ils vivront, ils fréquenteront les galeries. (Événements 44) 
Cette absurdité de «la force de l'habitude » résonne dans toute l'œuvre de Bernhard. Pour habiter un lieu, il faut y être habitué, avoir ses habitudes; ne pas être dérangé de l'extérieur, ou de l'intérieur. Perturbation va poursuivre cette réflexion sur l'habitat, en présentant une suite de lieux d'habitation spécifiques, au cours de la tournée d'un médecin, père du narrateur, des lieux bien sûr marqués par la maladie ou le mal-être, le meurtre ou le suicide : une auberge (où un crime vient d'avoir lieu), la maison d'un agent immobilier («vivant à Stiwoll, dans cette sorte «d'atroce enfer privé sous l'Alpe de Glein » qu'il s'était fabriqué lui-même » (Perturbation 33)), une chambre de malade ( « ma chambre à coucher est devenue ma chambre mortuaire » (Perturbation 43)), le pavillon de chasse d'un industriel (cloîtré dans sa pièce de travail), un moulin près d'une gorge (avec une cage à oiseaux exotiques, la plupart récemment massacrés), et enfin le château de Hochgobernitz du prince Saurau, lieu qui concentre toutes les réflexions de son propriétaire. Ce récit, où chaque lieu de vie se renverse sans cesse en un lieu de mort, où tout habitat est marqué par des catastrophes, apparaît comme un recueil de situations et de réflexions que toute l'œuvre ultérieure développera.

Il convient cependant de préciser qu'entre Amras et Perturbation, en janvier 1965 exactement, a lieu un événement majeur dans la vie de Bernhard : l'achat d'une propriété, la ferme d'Ohlsdorf à Obernathal, près de Gmunden ${ }^{4}$. «Une ruine », disait-il, dont il fait alors sa résidence principale; mais il faut effectuer de nombreux travaux de réfection afin de restaurer et de réhabiliter ce bâtiment du $\mathrm{XIV}^{\mathrm{e}}$ siècle, dix ans de travaux auxquels Bernhard lui-même participe activement.

Ma maison est à vrai dire une prison géante.

J'aime beaucoup cela; autant que possible, des murs nus. Nus et froids. C'est d'un très bon effet sur mon travail; mes livres ou les choses que j'écris sont ainsi, pareils à mon logis. (Trois jours 63)

Toute son œuvre - jusqu'à son écriture - s'en trouve imprégnée : outre Perturbation, qui réinvestit à vif des affaires personnelles liées à cette acquisition, La Plâtrière, Corrections, Oui, Béton ou Extinction déclinent alors toute une poétique de l'habitat. Oui reprend d'ailleurs, en la personne de Moritz (c'était déjà le Bloch de Perturbation), le personnage de son ami agent immobilier auprès de qui il avait acheté la ferme d'Ohlsdorf, une propriété qui correspond à celle décrite dans le récit :

En vérité, ma maison, dans l'état où je l'ai achetée, n'était rien d'autre qu'un toit troué, presque entièrement pourri, posé sur des murs croulants, bien qu'énormes. Mais j'étais assez jeune pour rendre cette ruine habitable, je m'étais fixé pour but de faire de cette ruine une maison d'habitation, en l'espace d'un an, de mes propres mains. (Oui 62)

Cet achat, et les activités manuelles qui lui sont liées, introduisent dans l'œuvre de Bernhard une dimension nouvelle : la construction (der Bau). Il ne s'agit pas seulement d'habiter un lieu, mais de le rendre habitable, d'en construire les conditions d'habitation. C'est pourquoi Corrections ou Oui développent largement les figures d'architectes et leur projet : un «Cône 
d'habitation », en ce qui concerne Roithamer, et une «villa de béton » en ce qui concerne le Suisse. Mais dans les deux cas, ces constructions, qui doivent accueillir un être particulier, une sœur ou une épouse, se révèlent être des tombeaux inhabitables, dans (ou devant) lesquels les destinataires de ces supposés lieux de vie se suicident. Il faut cependant reconnaître que la maison, dans laquelle on vit ou que l'on projette de construire, devient une obsession des récits de Bernhard.

Dans sa monographie sur Bernhard, Chantal Thomas reconnaît que la maison apparaît comme un personnage à part entière de l'œuvre de l'écrivain autrichien (cf. Thomas 14). Il convient toutefois d'élargir le terme à toute forme d'habitat, Bernhard s'intéressant à la capacité ou à l'incapacité de l'être humain à habiter un lieu. La «maison » se décline ainsi en différents lieux, bâtiments ou propriétés, dont la caractéristique principale, a priori, est d'être habitable : une auberge, une ferme, un château, une grande propriété familiale avec ses terres, mais aussi une tour, une cave, une galerie anti-bombardement, un magasin, un musée, un hôpital, un hospice, un asile ou une prison - voire un cimetière. Les personnages de Bernhard sont ainsi pris dans un lieu spécifique qu'ils sont censés habiter (ou fréquenter habituellement), mais qui s'avère rapidement un lieu impossible qui fait de son locataire un prisonnier. L'habitat devient vite, en effet, un lieu d'enfermement, froid et ténébreux, un endroit qui ruine son habitant, l'acculant à la folie, à l'asphyxie, à la maladie mortelle, au meurtre ou au suicide. « À la longue, je me suis complètement fourvoyé dans cette tombe qu'est ma maison. Je me lève le matin dans la tombe et, toute la journée, je cours de-ci de-là dans la tombe et, tard dans la nuit, je me couche dans cette tombe. » (Béton 23) Pourtant, l'habitat est nécessaire; et comme l'écrivain qui choisit de s'isoler dans une ferme à la campagne, les personnages principaux de Bernhard éprouvent toujours la nécessité d'un isolement radical, car leur travail les oblige à une telle retraite. Travailler - écrire, construire, penser - n'est possible que dans certaines conditions, parmi lesquelles le lieu joue un rôle primordial ou, pour mieux dire encore, central: il reste l'expression du «centre géométrique exact » (Corrections 310) qui donne un sens (en tout cas un lieu) au non-sens de l'existence. Mais de même que Bernhard ne pouvait véritablement travailler dans sa ferme ${ }^{5}$, ses personnages constatent que l'endroit choisi et consacré au travail s'avère être le lieu où le travail est impossible. Acheter la Plâtrière était pour Konrad devenu une nécessité, la «possibilité aussi d'y travailler intellectuellement »; mais le lieu s'avère inhumain, impossible à supporter pour autrui - pour Mme Konrad -, poussant Konrad lui-même à la démence et au meurtre. «Bien sûr, la Plâtrière est une prison, avait dit Konrad à Fro. Déjà de l'extérieur, elle donne l'impression d'une prison. Une maison de correction, un pénitencier, un bagne. » ( $L a$ Plâtrière 20) De Perturbation à Extinction, on passe ainsi souvent d'une volonté d'habitation et de construction à une volonté de destruction et de liquidation. Si le choix de «la Plâtrière » se révèle catastrophique, la construction du cône d'habitation, à laquelle Roithamer consacre sa vie, entraîne le suicide de la sœur chérie à qui le Cône était destiné; l'impossibilité de travailler dans la maison, avec ou sans la sœur, entraîne la fuite et la révélation du « Béton » comme symbole de l'absurdité et de l'horreur de l'existence; Extinction, enfin, clôture l'œuvre sur le mode d'une liquidation générale des propriétés familiales ${ }^{6}$. Comme le narrateur-personnage de Oui, on assiste à un mouvement contradictoire, un mouvement de va-et-vient qui peut être fatal. Le personnage affirme tout d'abord la nécessité de s'isoler dans un lieu de travail et de pensée, un «Arbeitskerker» ou une «Denkkammer», car il s'agit de mener à bien un travail de recherche et de réflexion (scientifique, intellectuel, artistique, philosophique) qui ne nécessite aucune 
intrusion extérieure, aucun contact. Mais au bout d'un certain temps, un tel isolement conduit à l'impossibilité de toute étude et de toute concentration, il conduit même à la folie et à la nécessité impérative de retrouver des «contacts »; le narrateur-personnage se réfugie chez son ami Moritz et y rencontre la Persane, avec qui il se promène, parle et se sauve de sa folie. Mais la rencontre épuise à son tour ses possibilités et déçoit chacun des protagonistes; c'est alors l'occasion pour le narrateur de rentrer chez lui et de retrouver son travail : il est à nouveau capable de lire et d'étudier, capable d'habiter sa «pièce aux livres » - quand la Persane, elle, se suicide dans sa «villa de béton».

Cette structure de va-et-vient parcourt toute l'œuvre de Bernhard: une situation donnée, d'autant plus quand elle est liée à l'immobilité d'un lieu, est une situation mortelle si on ne lui insuffle du mouvement. Deux solutions s'offrent ainsi à l'habitant dont le lieu d'habitation s'avère «catastrophique »: fuir, partir, marcher, se promener; ou liquider la «maison », la vendre, s'en séparer. Mais la fuite ou la marche peuvent à leur tour mener à la folie ou au suicide; car si elles permettent la parole et la pensée, elles entraînent immanquablement un épuisement, une exténuation, une déception. C'est ce que développe un récit comme Marcher (Gehen) : le danger du lieu, la nécessité de partir, l'impossibilité de véritablement partir, le «continuel va-et-vient (Hinundhergezogenwerden) des possibilités », l'épuisement de celles-ci. Si le récit met en évidence la «constante relation de confiance» qui existe entre la marche et la pensée, il insiste aussi sur le décalage intrinsèque à ces deux activités. La pensée ne connaît ni la distance, ni la vitesse; il s'agit toujours pour elle de s'exercer au sein d'un lieu circonscrit, afin de ne pas succomber à la folie, ou de limiter un épuisement qui semble pourtant inévitable. La fameuse «mansarde Höller » de Corrections, cette Denkkammer ${ }^{7}$ aux exigences les plus hautes, restera l'exemple de ce lieu de pensée idéal, sorte de lieu-cerveau à la mesure de ces « hommes de l'esprit» chers à Bernhard ${ }^{8}$. Mais cet endroit enfin habitable n'est-il pas aussi un lieu de destruction, selon le mouvement bernhardien du renversement catastrophique qui semble décidément inévitable ? N'est-ce pas là que l'œuvre de Roithamer a été à la fois possible et impossible, construite et déconstruite ? Les mouvements exposés dans Corrections, comme dans Le Naufragé (qui en est comme une reprise, une variation) ou dans Le Neveu de Wittgenstein ${ }^{9}$, creusent l'œuvre d'un mouvement sur place qui mène à la folie car rien ne peut l'arrêter. La prose prolifère sans qu'on puisse jamais en arrêter une proposition et la tenir pour vraie habitable. C'est le principe de Corrections, dans lequel vient se perdre toute l'œuvre de Bernhard : un processus de destruction, par corrections successives, réduit l'œuvre à néant. Mais ce processus lui-même, dans son écriture (même corrective), produit quand même, malgré tout, l'œuvre d'un tel désœuvrement; une œuvre se construit et s'offre au lecteur, au moins pour un temps, comme un lieu de parcours, et peut-être d'habitation.

\section{Le syndrome du terrier}

Le thème de la maison, de l'habitation chez Bernhard se confond ainsi souvent avec celui de l'œuvre elle-même, en train de s'écrire, par le truchement d'une œuvre fictive, celle sur laquelle butent les intellectuels des récits. Souvent, c'est un tiers (un ou plusieurs témoins) qui rapporte les propos d'un « homme de l'esprit », qui les recueille pour en proposer un ouvrage. L'écrivain met ainsi en scène une narration souvent réduite à un long soliloque, à une suite de propositions qui trouvent là le lieu de leur inscription. Il construit une «maison» à partir de propos qui 
peuvent s'annuler et se corriger les uns les autres; il invite à les parcourir, jusque dans leurs contradictions. Il s'agit d'apprendre à marcher dans une telle construction, d'apprendre à penser.

Celui qui pense conçoit d'ailleurs également sa pensée comme une marche, dit Oehler. Il dit : la démarche de mes, de ses ou de ces pensées. Il est donc parfaitement juste de dire : entrons dans cette pensée, comme si nous disions: entrons dans cette maison inquiétante. (Marcher 428)

Nous sommes ainsi invités à entrer dans « la Plâtrière », l'œuvre comme le lieu d'habitation, ce Kalkwerk, et d'en faire l'expérience labyrinthique. De phrase en phrase, nous voyons l'œuvre se construire, comme nous assistons à sa déconstruction. Chaque proposition rapportée est reprise, soulignée, parfois contredite; chacune renvoie à l'impossibilité d'une œuvre (l'Essai sur l'Ouïe auquel travaille Konrad) comme à l'impossibilité d'habiter un lieu, de même que chacune pose les pierres d'une œuvre qui se construit malgré tout, sur les ruines de l'autre. Ce paradoxe d'une œuvre qui se fait tout en se défaisant (où c'est le va-et-vient de la contradiction qui produit l'œuvre - qui la construit), nous semble refléter un héritage littéraire singulier, celui de Franz Kafka et tout particulièrement de son récit tardif intitulé Le Terrier, en allemand: Der Bau. L'œuvre de Kafka questionne elle aussi l'architecture (La Muraille de Chine) et les lieux de vie, les difficultés d'habiter un lieu, d'y travailler et d'y accepter autrui (du Verdict au Château); son écriture, en ses longues phrases labyrinthiques se renversant souvent sur elles-mêmes, présente également une architecture singulière qui entraîne son lecteur comme Joseph K. dans les dédales du Procès. Le Château, en allemand Das Schlo $\beta$, peut se traduire par « la serrure » : il s'agit bien d'essayer d'entrer dans le mystère de la construction, de la succession des pièces, d'arpenter l'espace dans le but, peut-être, d'habiter enfin. Mais Le Terrier nous semble encore plus explicite de ce geste d'écriture qui fonde toute une modernité et que nous retrouvons chez Bernhard. Der $B a u$, qui signifie d'abord et littéralement la construction ${ }^{10}$, avant de désigner un terrier ou une tanière, est un récit ou un rapport (Bericht) fait (construit) par un narrateur-personnage, que l'on peut identifier comme une bête, sur son existence au sein de sa «construction ». La bête est l'auteur de cette œuvre, et elle en est visiblement fière. " J'ai organisé mon terrier (ich habe den Bau eingerichtet) et il m'a l'air bien réussi (er scheint wohlgelungen). » (Kafka 1948, 115 et 1970, 359) Mais cette proposition, qui lance le récit ou la confession, sera constamment remise en cause jusqu'à ce qu'un « léger sifflement » se fasse entendre et confirme la menace. La vie du terrier (de la construction) repose entièrement sur sa sécurité : toute intrusion extérieure serait fatale à ce qui est conçu comme une «citadelle » ou un «château fort ». Ce récit, comme son titre l'indique, traite du problème de l'habiter, du point de vue du constructeur lui-même; on n'habite que ce que l'on a véritablement construit, de ses propres mains. Le pire des ennemis, selon la «bête ", serait d'ailleurs « quelque personnage de ma sorte, un connaisseur du bâtiment, quelque frère de la forêt, un amateur de vie paisible, mais une féroce canaille qui veut habiter sans bâtir » (Kafka 1948, 133). Les conditions de l'habitat, censées garantir à son habitant une protection sans faille, sont énoncées et niées les unes après les autres, ce qui a pour conséquence d'ébranler la construction elle-même; la possibilité d'un habiter est constamment menacée de se renverser en impossibilité. La présentation du terrier par son auteur déconstruit finalement 
l'édifice de sécurité qu'il était censé représenter. Ce mouvement catastrophique et ruiniforme loge dans le récit de l'habitant-(dé)constructeur; il en est le principe d'écriture.

Il n'est peut-être pas nécessaire d'insister sur les liens qui unissent le récit lui-même avec les conditions de sa production; il est bien évident que Der Bau est aussi la mise en abîme de l'écriture elle-même, d'une œuvre qui se compose dans l'insécurité. Rappelons que Kafka écrit ce texte alors qu'il est très malade et qu'il sait qu'il n'en a plus pour longtemps. Comme le précise Ernst Pawel, «ce fragment [est] le brillant autoportrait d'un homme enterré si bas qu'il n'est plus un homme, le reclus "emmuré dans la chambre la plus reculée d'une vaste cave", qui s'est creusé un abri pour se procurer au moins une illusion de sécurité » (Pawel 456). Nous retrouvons dans le récit ce paradoxe d'un habitat qui s'avère être un lieu d'enfermement. L'expression der Bau, d'ailleurs, signifie en argot la tôle, la prison. Autre rapport avec la situation de Kafka : le «léger sifflement» qui vient finalement troubler le calme relatif de la bête, sapant toute illusion de sécurité et de protection, n'est-il pas ce sifflement respiratoire que les poumons malades de l'écrivain produisent continuellement, annonçant sa mort prochaine ? Rappelons que Bernhard lui-même fut très tôt atteint par une maladie chronique des poumons qui feront de son «souffle », tout au long de sa vie, le témoin fragile d'une mort annoncée. Que faire contre ce compte à rebours? Parler, écrire, construire, penser. Être contre les faits, au moins contre ce fait-là. Mettre en place un discours - un souffle - interminable, qui épuise ses propres possibilités tout en laissant la trace de son épuisement. La «bête » de Kafka sait qu'elle n'en a plus pour longtemps, que sa construction est à chaque instant menacée de la destruction la plus impitoyable; mais contre ce fait, le récit doit avoir lieu: il doit même être interminable labyrinthique.

Le récit se présente en effet comme un labyrinthe, comme ce «labyrinthe de boyaux déconcertants », ou ces «labyrinthes zigzags » qui apparaissaient à leur auteur comme «le summum de l'art en matière de terriers (de constructions) » (Kafka 1948, 124-5). Le labyrinthe serait cette forme architecturale la plus à même de protéger son habitant, et de lutter contre toute intrusion extérieure. Le texte de Kafka se présente d'ailleurs comme un tel labyrinthe : loin d'être unilatéral ou linéaire, il s'élabore en plis et replis, en retours sur lui-même, en corrections. Les propositions associent sans cesse une affirmation à sa négation, à tel point que la «construction » du texte n'est plus qu'une suite de «déconstructions », installant ce paradoxe au cœur même du processus créatif : l'œuvre s'élabore comme désœuvrement. Le récit apparaitrait comme une suite de corrections ${ }^{11}: \mathrm{j}$ 'avance une proposition, « j'ai organisé mon terrier et il m'a l'air bien réussi », et je la corrige, car aucune proposition n'est absolument vraie. Une telle correction (un tel principe de correction) ne cesse ainsi de menacer l'œuvre en cours. Le texte progresse en reprises correctives qui vont souvent jusqu'à renverser (inverser) ce qui semblait acquis et venait d'être affirmé. Tout est passé au crible d'un renversement permanent, d'une autocorrection destituant toute assurance : l'ami désiré peut s'avérer être l'ennemi fatal (comme l'ennemi supposé peut se révéler être un ami), le dedans et le dehors, la sécurité et le danger, le propre et l'impropre se plient ou se renversent sans cesse l'un sur l'autre, au gré d'un discours soumis à la contradiction d'une illusion, d'un Schein. Dès la première phrase, la satisfaction du constructeur laisse échapper la crainte d'une faille irréparable : «er scheint wohlgelungen » - il semble, il a l'air, il est apparemment bien réussi. La réussite peut n'être qu'illusoire; les apparences peuvent être trompeuses. Le verbe scheinen joue sur l'évidence de l'éclat et la brillance (la vérité), comme sur l'illusion et le faux-semblant (le mensonge). La question de la 
ruse, intentionnelle ou non, et celle du crédit à porter au récit, s'imposent alors en donnant la tonalité (paradoxale) de toute la narration. Et ce mouvement catastrophique s'inscrit dans la phrase kafkaïenne, qui tisse ses affirmations et ses correctifs comme autant de touches, de coups de crayon. C'est la phrase elle-même qui se décompose et se rature (la ponctuation et la syntaxe jouent alors un rôle graphique essentiel) :

À quelque mille pas de là se trouve cachée, sous une couche de mousse qu'on peut relever, la véritable entrée de mon habitation $(B a u)$; elle est aussi bien défendue qu'une chose puisse l'être en ce monde : évidemment, quelqu'un peut marcher sur la mousse, on peut la crever d'un élan, et voilà mon entrée ouverte (dann liegt mein Bau frei da), et, si on veut - à conditions de posséder évidemment certaines qualités assez rares -, il n'y a plus qu'à entrer et à saccager tout (für immer alles zerstören). (Kafka 1948, 115-16)

Ou alors c'est l'enchaînement des phrases qui fonctionnent comme des touches qui viennent corriger les précédentes : «Mais le plus beau, dans ce terrier, c'est son silence. Évidemment, il est trompeur. Il peut se trouver soudain rompu et alors ce sera la fin de tout. Mais en attendant j'en jouis. » (Kafka 1948, 118) ${ }^{12}$ Il faut souligner l'importance des connecteurs logiques qui déplacent sans cesse le point de vue du discours, et le crédit qu'il se porte. Dans tous les cas, la place et l'ordre des mots, leur agencement, peuvent à tout moment déborder l'assurance du discours et ruiner une affirmation. «Das schönste an meinem Bau ist aber seine Stille » : la place de la conjonction, qui ici sert à coordonner cette affirmation avec la conclusion du paragraphe précédent, inscrit l'opposition dans la proposition elle-même, et annonce en quelque sorte le correctif négatif qui va bientôt être apporté à cette affirmation superlative.

L'œuvre s'élabore en même temps qu'elle s'efface, par ses ratures incessantes, par le mouvement ruiniforme qu'elle instaure et qui se déprend de ce sur quoi elle avait installé l'illusion de donner prise. Le donné est aussitôt repris. Mais si le récit se construit tout en se déconstruisant, affectant ses propres possibilités, il déjoue également les pouvoirs du lecteur. Celui-ci peut en effet apparaître comme cet autre du texte, qui vient sans cesse le menacer d'une visite. Une telle intrusion peut apparaître comme un bouleversement, un empiètement ou une dévastation, qui vient défaire ce qui a été laborieusement construit, qui vient fragiliser d'un seul coup ce qui s'est longuement et patiemment élaboré en vue de durer. Le lecteur est peut-être un tel prédateur qui affole le récit, et contre lequel le texte doit se défendre. Cette défense consiste à piéger le lecteur au moyen d'énoncés contradictoires, mais aussi à l'épuiser en exagérant les énoncés que l'on finit par raturer. Ce que la rature (la correction) atteint le plus profondément, ce n'est en effet pas tant l'écriture qui ne cesse alors de recourir à des stratégies d'auto-correction dans un mouvement infini, que la lecture qui dès lors s'épuise à se raccrocher à un énoncé qui puisse tenir. D'où le caractère inachevé, car interminable, de l'« œuvre » (de la « construction »).

Comme dans Le Terrier, les récits de Bernhard s'efforcent d'être imprenables, en reposant sur une topographie critique, voire aporétique. Ce sont des forteresses qui mettent en place un discours qui se contredit, qui dit contre (et qui exagère cette position, la pousse à bout, ad absurdum), comme s'il voulait que l'autre n'y trouve aucune entrée - ou bien qu'une fois entré, 
cet autre n'y trouve aucune sortie. Le médecin et son fils, qui rendent visite au prince Saurau, n'entreront jamais dans le château (et le texte y insiste) : ils restent sur le seuil, sur le mur extérieur de la propriété, à la fois dedans et dehors. Si l'ensemble des proses de Bernhard semble garder un rapport très fort avec le texte kafkaïen, La Plâtrière apparaît même, par certains aspects, comme une réécriture du Terrier. Nous retrouvons en effet dans ce roman certaines caractéristiques du récit de Kafka, et avant tout la figure centrale d'une construction/habitation, à savoir cette "Plâtrière » éponyme, ce Kalkwerk ou cette œuvre de plâtre : un lieu de travail, de solitude et de sécurité, qui devient (qui se renverse en) un lieu où le travail est impossible, où la solitude est constamment rompue par d'incessantes intrusions et où le crime est la réponse à une permanente insécurité. Le plus beau, dans cette "Plâtrière », c'est son silence. Évidemment, il est trompeur. Il peut se trouver soudain rompu et c'est la fin de tout. Tout le roman tourne en effet autour de l'élément sonore (l'Essai sur l'Oü̈e, l'extrême sensibilité auditive de Konrad, les exercices phonétiques et la nécessité de l'isolement pour préserver le silence) et de l'élément sécuritaire : Konrad était armé, lui un être «pusillanime» mais «devenu craintif », car il «voulait préserver la Plâtrière contre toute effraction, en particulier contre ce qu'on appelle les «éléments étrangers »... » (La Plâtrière 9) Dès que les conditions de travail sont réunies, et la Plâtrière est en elle-même un tel «centre géométrique exact », tout se disloque et se détruit. Il suffirait à Konrad d'écrire la première phrase de son essai pour que soit achevé ce traité qu'il a entièrement dans sa tête mais qu'il ne parvient pas à coucher par écrit; malheureusement, c'est toujours à ce moment là que l'on frappe à la porte ou que l'on fait du bruit à l'extérieur : tout le travail est à recommencer, pire : tout est détruit. En un instant, tout est possible et réalisable tout l'essai pourrait être écrit «d'un seul trait » : en un instant, tout peut être détruit. C'est quand Konrad ne travaille pas que règne dans la maison le calme le plus «absolu »; c'est quand il se met au travail que le calme est rompu... ${ }^{13}$

Tout se renverse en son contraire selon une construction paradoxale qui fait de la destruction un élément essentiel de cette construction. C'est ce que souligne également Aldo Gargani, attentif au motif du «va-et-vient» (Hinundhergezogenwerden) ou à celui du « d'une part-d'autre part»(Einerseitsandererseits) qui caractérisent selon lui «la phrase infinie de Thomas Bernhard » :

L'écriture de Bernhard est la découverte des aspects contrastés et paradoxaux des faits. Le chemin radical qu'elle emprunte est celui d'une textualité prompte à renverser toute phrase en son contraire, une textualité qui s'expose à chaque instant à sa propre négation, car au terme de ce parcours paradoxal, une telle écriture est destinée à montrer quelque chose qui ne lui appartient pas et vers quoi elle penche, en quelque sorte, hors d'elle-même. (Gargani 13)

L'écriture de Bernhard met à nu l'absurdité des propositions figées en en exagérant la contradiction. Elle révèle «la part de vérité qu'il y a dans le mensonge » (La Cave 132) en s'érigeant comme résolument critique. C'est une écriture contre, ou une contre-écriture, proche de ce que le poète Paul Celan a nommé une Gegenwort, une contre-parole (Celan 63). Le livre n'est-il pas, dès lors, le lieu extrême - utopique ? - où se réalise un tel projet critique ? Que reste-t-il, au terme d'un tel processus de contradiction et d'effacement (d'Auslöschung, ultime 
ouvrage de l'auteur sous-titré : ein Zerfall, soulignant le délitement progressif, le déclin, l'éboulement, la décomposition), si ce n'est l'ouvrage lui-même qui, ultime paradoxe, sauve l'œuvre d'une extinction définitive ? Encore faut-il lire un tel ouvrage, c'est-à-dire en réactiver le processus de délitement.

\section{Construire / déconstruire le livre : une hospitalité négative}

C'est peut-être avec Corrections que Bernhard pousse jusqu'au bout le principe d'écriture kafkaïen. C'est d'abord un récit dont le personnage central, Roithamer, consacre sa vie à une construction, un cône d'habitation (Kegelbau) destiné à sa sœur, un projet né de l'observation de la construction de la maison Höller. «Que le terme construire (das Wort Bauen) était l'un des plus beaux qu'il soit, nous le savions depuis que Roithamer avait parlé sur ce sujet» (Corrections 19-20). Il est en effet question de « construction », d' « art de la construction », mais pas d' « architecte » ou d' « architecture », termes abhorrés, absolument rejetés ${ }^{14}$. Son autre projet est un essai sur Altensam, la maison familiale dont le concept, selon Roithamer, était l'anéantissement total de sa personne (cf. Corrections 259). Le narrateur, ami du défunt Roithamer, doit «mettre en ordre et trier» les papiers laissés par son ami, relativement à ces deux projets d'écriture. Il met à nu une singulière conception de l'œuvre, que développe et réalise le récit lui-même : une «construction» par corrections successives, un «processus de renversement incessant de toutes ses pensées à l'intérieur de l'ensemble » (« en retournant son sens par une correction totale » Corrections 99), jusqu'à l'effacement complet de l'œuvre ellemême :

je pensais comme je peux en juger à ses corrections que c'était par ce processus de ne plus laisser subsister d'un tout comprenant plus de huit cents pages, qu'un tout de seulement quatre cents pages et ensuite qu'un tout de cent cinquante pages, puis seulement de quatre-vingt pages puis finalement de même pas vingt pages et même dans une ultime conséquence de ne plus rien laisser subsister de l'ensemble - que c'était seulement par ce processus que l'ensemble était né; tout cela réuni constitue le tout, me dis-je. .. (Corrections 203)

Il s'agit de construire une œuvre par destructions successives : le processus de corrections qui aboutit à un épuisement total des possibilités de l'œuvre est paradoxalement ce qui construit cette œuvre. Qu'est-ce qu'une écriture raturée, corrigée jusqu'à l'effacement ? Quel est cet « art de la prose (Prosakunst) » propre à Roithamer, qui adopte un principe de renversement catastrophique (jusqu'à l'anéantissement) en guise de processus créateur? "Toute correction est destruction, anéantissement, selon Roithamer. »(Corrections 403) Elle aboutit à la correction suprême, le suicide de son auteur, mais cet acte ultime laisse les traces du processus de correction qui est un processus de création, même si c'est une création par destructions successives. Car les notes ont été recueillies, comme la parole de la bête a été consignée dans un ouvrage. L'écriture garde les traces des corrections successives, elle inscrit le soliloque dans un lieu qui délimite le processus sans l'arrêter. Le livre apparaît peut-être, en fin de compte, comme le dernier lieu habitable, 
comme cette «clairière (Lichtung)» qui clôture Corrections. «Das Ende ist kein Vorgang. Lichtung. » (Korrektur 463) ${ }^{15}$ La fin n'est pas l'accomplissement du processus de destruction : elle éclaire en retour tout ce qui a été inscrit (et peut-être lu), met en évidence l'œuvre qui s'est agencée par destructions successives mais contre tout anéantissement définitif.

L'expérience kafkaïenne, dont Le Terrier présente le syndrome et que l'œuvre de Bernhard poursuit et exagère, ne cesse de poser une question : que reste-t-il au terme de cette constructiondestruction qui épuise les assurances et déjoue les habitudes (d'écriture comme de lecture) ? Il reste un texte où l'objet littéraire et littéral est porté à ses limites explosives, où les significations s'épuisent, où la ruine gagne chaque lieu, chaque moment de la construction $(\mathrm{Bau})$. Il reste un dessin d'écriture dont l'autocorrection permanente frappe l'œuvre d'une sorte de pulsion de mort qui fait prendre des décisions contraires à l'intérêt de départ. Le texte garde en lui-même cette capacité mystérieuse qu'ont les organismes biologiques de se détruire, mais aussi de se protéger contre cette autodestruction, selon un principe d'auto-immunité ${ }^{16}$ qui est peut-être le signe d'une vie de l'œuvre, que chaque lecture réactive.

L'écriture de Bernhard participe d'un dessin d'écriture que, dans Perturbation, le prince Saurau nomme un «enchevêtrement de lignes » (Liniengewirr). Cet enchevêtrement est le résultat de différentes strates de destructions paradoxalement érigées en construction. "Chaque jour, je me construisais totalement puis me détruisais totalement. » (Perturbation 49) Après le peintre Strauch de Gel, l'industriel du pavillon de chasse, dans Perturbation, s'ajoute à la longue liste de ces créateurs/destructeurs dont les œuvres sont le résultat d'une impossibilité ou d'une destruction. «Il travaillait nuit et jour, écrivait puis détruisait ce qu'il avait écrit, écrivait encore et détruisait encore et encore, et se rapprochait de son but. » (Perturbation 52 et cf. 59) À la liquidation de la propriété familiale de Wolfsegg (Extinction) répond la destruction des écrits du « penseur détesté » par sa propre famille; mais c'est le plus souvent le « penseur » lui-même qui conçoit son œuvre comme une suite de destructions, ou tout au moins une suite de corrections qui aboutissent à l'anéantissement de l'œuvre. « Correction de la correction de la correction de la correction, selon Roithamer. » (Corrections 408) Le Naufragé, ou le «sombreur », présente ces deux types de création par destruction: Wertheimer laisse, en se suicidant, des écrits que la famille se chargera de détruire ${ }^{17}$; le narrateur, quant à lui, s'efforce d'écrire un essai sur Glenn Gould, multipliant les versions, les corrections et les destructions.

J'avais finalement derrière moi huit tentatives de cette sorte qui se sont toutes soldées par la destruction des esquisses quand enfin, à Madrid, je sus comment aborder mon écrit Sur Glenn que j'ai ensuite d'ailleurs mené à bonne fin dans mon appartement de Calle del Prado, pensai-je. Mais déjà, j'étais de nouveau dans le doute, je me demandai si cet écrit avait effectivement une valeur quelconque et songeai à le détruire à mon retour; tout écrit, pour peu que nous le laissions reposer un certain temps et le reconsidérions encore et encore depuis le début, finit par nous paraître insupportable et nous n'avons de repos que nous ne l'ayons détruit, pensai-je. (Le Naufragé 86) 
N'oublions cependant pas l'ironie de Bernhard, ce sens du tragi-comique et son art de l'exagération. Exagérer son œuvre, c'est l'écrire par destructions successives, c'est l'ériger sur des ruines. La création est pour l'écrivain le signe d'un «monstrueux épuisement»: «die Schöpfung ist eine ungeheuere Erschöpfung » (Verstörung 40). Mais cette équation résulte d'un jeu de mots ${ }^{18}$, d'un maniement des préfixes; il suffit d'ajouter un préfixe à un mot pour que celui-ci se renverse en son contraire, ou se détruise - pour qu'il s'épuise. La langue de Bernhard apparaît en effet comme une tentative paradoxale de construction/destruction de et dans la langue, offrant et niant dans le même mouvement toute hospitalité. Phrase après phrase, reprenant, enlevant, rajoutant ou modifiant, l'écrivain bâtit son œuvre, sa maison littéraire, même si celle-ci tend à ne se réduire qu'à une mansarde donnant sur un précipice, ou à une cave dans laquelle menace sans cesse le risque d'un étouffement. Tout lecteur de Bernhard fait cette expérience singulière de constater, en lisant, que l'œuvre est en train de se construire en même temps qu'elle se défait sans cesse, sapant les fondations sur lesquelles elle était censée s'ériger.

Je suis un démolisseur d'histoires, je suis le démolisseur d'histoires type. Dans mes écrits, si une anecdote se dessine ou si seulement je vois de loin, derrière une colline de prose, apparaître le vague contour d'une histoire, je l'abats. De même les phrases, j'aurais envie de tuer des phrases entières dès que je vois qu'elles pourraient se former. (Trois jours 63)

Il est étonnant de lire ces lignes tout en pensant aux ouvrages de Bernhard, ces blocs de prose de plus en plus longs, contenant peu de chapitres. L'écriture de Bernhard est loin de révéler la blancheur de la page; il la noircit au contraire, la raye de ces «enchevêtrements de lignes » d'écriture où s'entremêlent différents types de constructions : des constructions d'habitats, des constructions de pensées et des constructions verbales. Bernhard construit de plus en plus souvent ses livres, au fil de son œuvre, sur un mode musical, les soliloques des personnages et (ou) des narrateurs étant rythmés sur des airs catastrophiques. Jubilation de la noirceur : noircir du papier, c'est chanter cette noirceur d'un souffle singulier, peut-être inaudible. Comme la Joséphine de Kafka, le chant de Bernhard n'en est peut-être pas un, et puis sa place au sein de la communauté est difficilement acceptable. Il reste un souffle singulier, ce souffle pour lui si précieux, comme l'était celui de Kafka. Continuer à produire des phrases, quitte à les détruire une fois proférées, c'est continuellement reprendre son souffle afin de construire sa maison-livre - un lieu qu'il nous échoit, à nous lecteurs, d'habiter un temps, difficilement, avant d'en être à notre tour rejetés, épuisés. On assiste en effet à un étrange mouvement paradoxal d'attirance dans l'habitation et de rejet hors de celle-ci, qui renvoie au mouvement de la lecture que Bernhard nous invite à suivre : entrer dans l'œuvre (croire que l'on va y trouver des vérités), en suivre le mouvement déceptif, pour en être finalement rejeté (ultime correction). Le livre de Bernhard n'est pas habitable, ou bien il l'est contre toute attente, parce qu'il est le lieu critique d'un rejet et d'une hospitalité infinie - ce que l'on pourrait désigner comme une hospitalité négative (comme l'on parle de théologie négative). 
Écrire, habiter, penser : on aura vu que ces trois activités sont nécessaires (not-wendig) mais qu'elles présentent un risque, celui d'une décomposition, d'une dégradation - l'épuisement d'un lieu, l'épuisement de la pensée : une exténuation. On n'en sort pas : habiter est une tâche à la fois nécessaire et impossible. Pourtant se dessine dans cette œuvre une sorte d'utopie salvatrice, une «tentative de sauvetage » (Rettungsversuch) ${ }^{19}$ que prend en compte l'écriture elle-même en tant qu'elle fait œuvre (jusque dans son désœuvrement), ou qu'elle se fait livre. Le souffle impressionnant et épuisant des phrases de Bernhard est bien un acte de création, relève bien d'un geste de construction, même si son matériau est le plus souvent négatif et si son processus d'élaboration s'appuie sur une «déconstruction». En allant au bout de ce processus, en l'exagérant, l'écrivain fait œuvre, c'est-à-dire qu'il invente un lieu d'écri-lecture, un « enchevêtrement de lignes » au fonctionnement infini - jamais habitable, à jamais habitable.

\section{Bibliographie}

Bernhard, Thomas. Gel, Paris, Gallimard, trad. par Boris Simon et Josée Turk-Meyer, 1967.

- Verstörung, Frankfurt am Main, Insel Verlag / Suhrkamp, 1967 / 1974.

---. La Plâtrière, Paris, Gallimard, trad. par Louise Servicen, 1974.

---. Korrektur, Frankfurt am Main, Suhrkamp, 1975.

---. La Cave, Paris, Gallimard « Biblios », trad. par Albert Kohn, 1976, 1990.

---. Corrections, Paris, Gallimard, trad. par Albert Kohn, 1978.

---. Oui, Paris, Gallimard, trad. par Jean-Claude Hémery, 1980.

---. Béton, Paris, Gallimard, trad. par Gilberte Lambrichs, 1985.

---. Le Neveu de Wittgenstein, Paris, Gallimard, trad. par Jean-Claude Hémery, 1985.

---. Trois jours, Paris, Maurice Nadeau, trad. par Jean De Meur in Ténèbres. Textes, discours, entretien,1986.

---. Amras et autres récits [dont le récit Marcher], Paris, Gallimard, trad. par Jean-Claude Hémery et Eliane Kaufholz, 1987.

---. Événements, Paris, L’Arche, trad. par Jeanne Étoré et Bernard Lortholary, 1988.

---. Perturbation, Paris, Gallimard, trad. par Bernard Kreiss, 1989.

---. Extinction, Paris, Gallimard, trad. par Gilberte Lambrichs, 1990.

---. Dans les hauteurs. Tentative de sauvetage, non-sens, Paris, Gallimard, trad. par Claude Porcell, 1991.

---. Entretiens avec Krista Fleischmann, Paris, L’Arche, trad. par Claude Porcell, 1993.

---. Récits 1971-1982, Paris, Gallimard «Quarto », éd. par Jean-Marie Winkler, 2007.

Celan, Paul. Le Méridien \& Autres proses, Paris, Seuil, trad. par Jean Launay, 2002.

Derrida, Jacques. Foi et savoir, Paris, Seuil, 1996, 2000.

Fontanier, Les Figures du discours, Paris, Flammarion, 1977.

Gargani, Aldo G. La Phrase infinie de Thomas Bernhard, Cahors, Éditions de l'Éclat, 1990.

Heidegger, Martin. «Bâtir habiter penser», in Essais et conférences, trad. par André Préau,

Paris, Gallimard, trad. par Jean-Pierre Cometti, 1958.

Höller, Hans. Thomas Bernhard - une vie, trad. par Claude Porcell, Paris, L'Arche, 1994.

Kafka, Franz. Le Terrierin La Colonie pénitentiaire et autres récits, Paris, Gallimard, , trad. par Alexandre Vialatte, 1948.

---. Der Bau, in Sämtliche Erzählungen, Frankfurt am Main, Fischer Verlag, 1970. 
Pawel, Ernst. Franz Kafka ou le cauchemar de la raison, Paris, Seuil, trad. par Michel Chion et Jean Guiloineau, 1988.

Thomas, Chantal. Thomas Bernhard, Paris, Seuil « Les Contemporains », 1990.

${ }^{1}$ Dans la monographie qu'il consacre à l'écrivain, Hans Höller parle d'un « complexe de l'origine » qui explique le fait que «dans les œuvres en prose qui viendront, c'est toujours dans des bâtiments que le moi reconstruira son histoire. » (Höller 31)

${ }^{2}$ Cf. Entretiens avec Krista Fleischmann 35 : «Tout est exagéré, mais sans exagération, on ne peut rien dire du tout, parce que dès que vous élevez simplement la voix, c'est déjà, en fait, une exagération, car pourquoi l'élevezvous ? Quand on dit quoi que ce soit, c'est déjà une exagération. Cette phrase est inattaquable. Vous ne pouvez rien avoir à redire à cette phrase. »

${ }^{3}$ Cf. Entretiens avec Krista Fleischmann 13 : «Je n'écris jamais que sur des paysages intérieurs. . Je crois que je n'ai encore, dans aucun livre, décrit de paysage. Rien de tout ça. Je n'écris jamais que des concepts, c'est toujours des montagnes ou une ville ou des rues, mais comment elles sont - je n'ai jamais fait de description de paysage. Ça ne m'a d'ailleurs jamais intéressé. » Ce refus de la description extérieure explique l'intérêt de Bernhard pour l'habitat ou tout lieu d'enfermement qui met l'accent sur les "processus intérieurs » dans lesquels les personnages sont pris. La Plâtrière, la mansarde Höller, Altensam, Wolfsegg, sont d'abord des noms, des lieuxconcepts.

${ }^{4}$ Si ce premier accès à la propriété restera déterminant pour l'évolution de son œuvre, il achètera plus tard deux autres demeures qui laisseront des empreintes dans ses livres : la «Krucka» à Grasberg (en 1971) et la « Hanspäun » (en 1972), située en lisière de forêt, à Niederpucheim près de Wolfsegg et d'Ottnang.

${ }^{5}$ Comme on peut le constater dans son cycle autobiographique ou dans les biographies qui lui ont été consacrées, Bernhard a toujours «bougé », et ce dès sa naissance dans une clinique de Hollande : de déménagement en déménagement, de voyage en voyage, sa vie n'a jamais été limitée à un lieu fixe (ni même à un pays). Bernhard écrivit d'ailleurs le plus souvent ses livres lors de ses voyages à l'étranger. Voir la notice biographique «Vie et œuvre de Thomas Bernhard » qui clôt le gros volume Quarto édité par Jean-Marie Winkler (Récits 1971-1982 nota. 908).

${ }^{6}$ Mais le principe de la liquidation est déjà à l'œuvre dans Perturbation, dans La Plâtrière ou dans Corrections : tout lieu d'habitation, tout lieu de vie est amené à se vider, à se défaire de toute attache, propulsant son habitant dans une solitude et un dénuement tout aussi terrifiants.

7 Il s'agit d'un jeu de mot construit à partir de Dachkammer, la mansarde, qui se révèle être une «Denkkammer », car «tout dans cette chambre (Kammer) se rapportait uniquement à la pensée (das Denken) » (Corrections, 29; Korrektur 23).

${ }^{8}$ Cf. Corrections 14 : «ici, dans la mansarde Höller, écrit-il, m'a été possible tout ce qui m’a toujours été impossible en dehors de la mansarde Höller »;

le plus incroyable n'était tout à coup plus incroyable dans la mansarde Höller, la chose la plus impossible (penser !) n'était plus impossible. Les conditions qui étaient le plus nécessaire à sa pensée et qui la favorisaient le plus, il les avait toujours trouvées dans la mansarde Höller; pour mettre en marche sans façons et avec une absence totale de perturbation le mécanisme de sa pensée il n'avait besoin que de se rendre dans la mansarde Höller, de quelque endroit que ce fût, et ce mécanisme fonctionnait. (Corrections 15)

${ }^{9}$ A titre d'exemple, voir Le Neveu de Wittgenstein 116-7 :

Tous mes ancêtres étaient possédés de cette même agitation, et ils ne tenaient pas en place, ni en un endroit, ni dans un fauteuil. Trois jours à Vienne et je n'y tiens plus, trois jours à Nathal et je n'y tiens plus. Pendant les dernières années de sa vie, mon ami s'était accordé à mon rythme d'allers et retours, et il m'avait souvent accompagné quand j'allais à Nathal, puis en revenais, et vice versa. . . Et, la vérité, c'est que je ne suis heureux qu'installé en voiture entre l'endroit que je viens de quitter et celui vers lequel je roule, je ne suis heureux qu'en voiture et pendant le trajet. . Je fais partie de ces êtres qui au fond ne supportent pas un endroit sur terre et ne sont heureux qu'entre les endroits d'où ils partent et vers lesquels ils se dirigent. 
${ }^{10}$ Der Bau n'est pas seulement le bâtiment, l'édifice, mais le processus même de la construction. Le «terrier » du narrateur de Kafka est bien une habitation prise dans un mouvement perpétuel de construction (améliorations, déplacements, travaux d'aménagement).

${ }^{11}$ Nous entendons la « correction » comme le principe général provenant de cette figure de rhétorique (cf. Fontanier 366) qui consiste à mettre en scène, dans un même discours, un énoncé et sa correction, autocorrection qui peut aller jusqu'à la négation même de l'énoncé. Cette sorte d'épanorthose, qu'on appelle aussi justement retouche corrective, contamine tout le discours kafkaïen, qui devient alors emblématique de cette perte des assurances qui catastrophie le propre discours d'un narrateur (de même que tout discours du propre). Inutile d'insister sur le fait que l'auteur de Corrections est l'héritier direct de ce principe d'écriture.

12 «Das schönste an meinem Bau ist aber seine Stille. Freilich, sie ist trügerisch. Plötzlich einmal kann sie unterbrochen werden und alles ist zu Ende. Vorläufig aber ist sie noch da. » (Kafka 1970, 361)

${ }^{13}$ Cf. La Plâtrière 65. Nous pourrions développer bien davantage les similitudes entre le roman de Bernhard et le récit de Kafka, mais cela nécessiterait une étude à part entière; seule nous importe ici la mise en évidence d'un même principe d'écriture.

${ }^{14}$ Cf. Corrections 19. Le texte allemand joue davantage sur les mots et oppose à Architekt ou Architektur divers substantifs construits à partir du radical Bau ou Bauen: «Baumeister», «Bauwerk» et «Baukunst» (cf. Korrektur 14).

15 «La fin n'est pas un processus. Clairière. » (Correction 410)

${ }^{16}$ Nous empruntons cette notion à Jacques Derrida, notion qui hante ses derniers livres depuis Foi et savoir.

${ }^{17}$ Le narrateur, comme celui de Corrections, voudrait récupérer « coûte que coûte » ces papiers sinon condamnés par la famille à la destruction. Cf. Le Naufragé 45.

18 On pense aux jeux de langage (Sprachspiele) d'un Wittgenstein. Un tel jeu sur les mots participe ici tout à la fois de la construction et de la destruction du langage.

${ }^{19}$ Cf. l'ouvrage de Bernhard écrit en 1959 mais qu'il n'a décidé de publier que peu de temps avant sa mort : Dans les hauteurs. Tentative de sauvetage, non-sens. 